COMMENT. The lenticular nuclei may contribute to the mechanism of infantile spasms. A cortical-subcortical interaction is proposed in the pathophysiology of infantile spasms. A Commission on Pediatric Epilepsy of the International League Against Epilepsy, following a workshop on infantile spasms, has proposed that the term infantile spasms is too restrictive and the term "spasms" is preferable to designate a special type of epileptic seizure that involves the axial musculature-in flexion, extension or mixed- and that often occurs in clusters. They propose that this type of seizure should be listed in the International Classification of Epileptic Syndromes (Commission 1981) and not be confined to the International Classification of Epileptic Syndromes (Commission 1989).

\title{
VALPROATE METABOLITES DURING TREATMENT OF INFANTILE SPASMS
}

Metabolite profiles were measured in serum and urine of pediatric patients treated with valproic acid for infantile spasms at the Institute of Toxicology and Embryopharmacology, Free University Berlin and Department of Pediatrics, Rittberg-Krankenhaus, Berlin, Germany. The mean age was 7 months (range 4-12 months) and all 25 patients had the hypsarrhythmia pattern in the EEG. Eighteen patients were seizure free after 3 months of VPA monotherapy in a dose of $100 \mathrm{mg} / \mathrm{kg}$ body weight/day. The main VPA metabolites in serum were the $\beta$-oxidation products (2-en-VPA and 3-ketoVPA) and the major diunsaturated metabolite 2,3'-dien VPA; 4 -en and 3-keto4 -en, 2 potential hepatotoxins, were detected only in very low concentrations. Glucuronide conjugates and the oxidation products represent the most abundant metabolites in urine. Two children had transient abnormal metabolite profiles indicating altered $\beta$-oxidation and associated with hepatomegaly and increased liver enzyme activity (Fisher E et al. Valproate metabolites in serum and urine during antiepileptic therapy in children with infantile spasms: abnormal metabolite pattern associated with reversible hepatotoxicity. Epilepsia Jan/Feb 1992; $\underline{33}: 165-171)$. (Reprints: Professor Dr. H. Nau, Institute of Toxicology and Embryopharmacology, Free University Berlin, Garystrasse 5 , D-1000 Berlin 33, Germany.)

COMMENT. The initial stages of hepatotoxicity reactions to VPA may be accompanied by characteristic changes in VPA metabolism (increased levels of 2-en, 2,3'-dien and 3-en VPA). The concentrations of unsaturated metabolites and enzyme activity became normal in 2 children on reduction of VPA dose and concomitant lowering of fever. These abnormal metabolite patterns occurred predominantly in patients treated with large doses of VPA combined with dexamethasone. The authors propose that the early detection of such abnormal metabolite patterns might decrease the risk of severe hepatic injury by the timely withdrawal of VPA or reduction of dosage.

\section{MRI IN TUBEROUS SCLEROSIS WITH NORMAL I.Q.}

The number, size and distribution of cerebral hamartomas determined by MRI in 11 patients with tuberous sclerosis and normal intellect are 
reported from the Bath Unit for Research into Paediatrics, Royal United Hospital, Combe Park, Bath, Avon, England and Bristol MRI Centre, Frenchay Hospital, Bristol. Nine patients had between 2 and 9 cerebral tubers seen on MRI T2 weighted images, 7 had 1-5 subependymal nodules and 2 had normal scans. The hamartomas were at least $4 \mathrm{~mm}$ in size. The number of lesions found on the MRI may not be used to predict neurological outcome in tuberous sclerosis. Only 3 of the 11 patients had a history of seizures and all had normal intellect (Webb DW et al. Cranial magnetic resonance imaging in patients with tuberous sclerosis and normal intellect. Arch Dis Child; Dec 1991; 66:1375-1377). (Reprints: Dr. Osborne, Royal United Hospital, Comb Park, Bath, Avon BA1 3NG, England.)

COMMENT. The authors suggest that the wrong conclusions may be drawn if the number of lesions alone on cranial MRI is used to predict neurological outcome in tuberous sclerosis. Lesions seen on both MRI and CT may become less obvious with advancing age in patients with tuberous sclerosis. In the experience of these authors, individuals who reach 5 years of age with normal development do not develop mental handicap from tuberous sclerosis. While the number of cerebral hamartomas appears to be an unreliable predictor of clinical outcome it is possible that the distribution and size of lesions may be more helpful. Patients with temporal lobe or occipital lobe hamartomas are most likely to have seizures or EEG abnormalities. Tuberous sclerosis was the cause of infantile spasms in $10 \%$ of cases treated in Finland (see Ped Neurol Briefs April 1990) but in only 2\% reported from China (Feng YK et al. Chinese Med J. May 1991; 104:416-421).

\section{ANTIEPILEPTIC DRUGS}

\section{VALPROATE FOR INTRACTABLE EPILEPSY}

The efficacy and side effects of high dose valproic acid (VPA) therapy in 46 children with refractory epilepsy were studied at the Department of Child Neurology, Okayama University Medical School, Okayama, Japan. Serum VPA concentrations ranged from 105.1 to $198.4 \mathrm{mcg} / \mathrm{ml}$. Seizures were completely controlled in $15(32.6 \%)$ and improved in $12(26.1 \%)$. This degree of control was sustained at 6 months or more follow-up. VPA therapy was especially effective for West syndrome and for epilepsy with continuous spike waves during slow wave sleep. Symptomatic generalized epilepsy and symptomatic partial epilepsy were benefitted equally. No significant difference in efficacy was noted between the monotherapy and bipharmacy groups, but adverse side effects appeared more frequently and higher serum levels of VPA were difficult to obtain in the bipharmacy group. Liver enzymes were transiently increased in $50 \%$ of the patients and elevated serum amylase occurred in $10 \%$ of patients. Hyperammonemia in $6.5 \%$ of patients necessitated withdrawal of the drug. Thrombocytopenia less than $100,000 / \mathrm{mm}^{3}$ occurred in $21.7 \%$ and hypofibrinogenemia was detected in $65 \%$ of patients. These side effects were reversible with reduction of dosage 